\title{
The Phenomenological Theory of Exchange Currents in Nuclei*
}

\author{
R. K. Osborn and L. L. Foldy \\ Case Institute of Technology, Cleveland, Ohio \\ (Received May 1, 1950)
}

\begin{abstract}
As was first pointed out by Siegert, the existence of exchange forces in nuclei implies the existence of accompanying exchange currents. Sachs has calculated an expression for these, by making the Hamiltonian containing exchange potentials gauge-invariant, and has applied it to the calculations of exchange magnetic moments in $\mathrm{H}^{3}$ and $\mathrm{He}^{3}$. The Hamiltonian obtained by Sachs is not the most general admissible one. More generally, the exchange current density is found to depend on a vector function whose irrotational part is completely determined by gauge-invariance but whose solenoidal part is arbitrary except for the requirements (following from conditions of translational invariance and symmetry in all nucleons on the Hamiltonian) that it be translationally invariant and antisymmetric under the exchange of the spin and space coordinates of each pair of nucleons. Making use of these
\end{abstract}

conditions on the Hamiltonian, the explicit form of the dependence of the solenoidal part of the exchange current upon the spin and isotopic spin coordinates of the nucleons has been derived. In the resultant exchange moments, the irrotational part leads to the expression obtained by Sachs, while the solenoidal term contribution contains the spin operators of the nucleons in particular combinations, together with arbitrary functions of the nucleon separation. Villars' exchange moment expression, as obtained from meson theory, is included as a special case and hence the exchange contributions to the moments of $\mathrm{H}^{3}$ and $\mathrm{He}^{3}$ are explicable on a phenomenological basis, contrary to the results obtained in Sachs' special case. The generality and significance of the results are discussed in relation to the various meson theories.

\section{INTRODUCTION}

$\mathrm{A}^{\mathrm{s}}$ $\mathrm{S}$ was first pointed out by Siegert, ${ }^{1}$ the existence of charge exchange forces between nucleons together with the differential conservation law for electric charge require that electric currents must flow in the intervening space between interacting neutrons and protons. These so-called exchange currents will then interact with electromagnetic fields and modify the electromagnetic properties of nuclei in two important respects. First, they will give rise, in general, to a contribution to the magnetic moment of a nucleus above and beyond those arising from the intrinsic magnetic moments of the constituent nucleons and from their orbital motion. Second, they will lead to a modification of the cross sections for most photo-nuclear processes. Recently considerable interest has been aroused in the effects of these exchange currents as a consequence of recent highly accurate measurements ${ }^{2}$ of the magnetic moments of $\mathrm{H}^{3}$ and $\mathrm{He}^{3}$ which appear to show definitely the existence of exchange contributions to these moments.

As Siegert also pointed out, the exchange current density required for conservation of charge is not completely determined by the exchange interaction itself. Only its irrotational (longitudinal) part is uniquely so determined while the solenoidal (transverse) part is arbitrary. Sachs ${ }^{3}$ has recently determined a particular exchange current distribution which satisfies the condition of charge conservation in which the exchange currents are assumed to flow along straight line filaments connecting the interacting nucleons. He applied his results to the calculation of the exchange contributions to the magnetic moments of $\mathrm{H}^{3}$ and $\mathrm{He}^{3}$ and found that this current distribution could not account

\footnotetext{
* This work has been supported by the AEC.

1 A F. Siegert, Phys. Rev. 52, 787 (1937).

2 H. L. Anderson and A. Novick, Phys. Rev. 71, 372 (1947); 73, 919 (1948). Bloch, Graves, Packard, and Spence, Phys. Rev. 71, 373, 551 (1947).

3 R. G. Sachs, Phys. Rev. 74, 433 (1948).
}

for the observed results. An examination of Sachs' results in the light of the present investigation shows that while his particular current distribution contains both an irrotational and a solenoidal part, only the irrotational part gives a contribution to the magnetic moment. One must therefore conclude that the major part of the exchange contribution to magnetic moments arises from the indeterminate solenoidal part of the exchange current distribution.

According to current views, the carriers of charge between nucleons are presumed to be the mesons responsible for nuclear forces. If these views are correct, the description of nuclear forces by any particular type of meson theory will lead to a unique expression for both the irrotational and solenoidal parts of the exchange current density and their contributions to the magnetic moments of nuclei. Villars ${ }^{4}$ has performed a calculation along these lines employing the symmetrical pseudoscalar meson theory and has found that the experimental results on the moments of $\mathrm{H}^{3}$ and $\mathrm{He}^{3}$ can be explained on this model; however, similar calculations on the basis of other meson theories (vector and

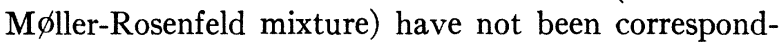
ingly successful. ${ }^{5}$ The well-known deficiencies of all forms of meson theory at the present time promote interest, accordingly, in determining what features of exchange currents in nuclei can be formulated independently of specific assumptions concerning the fieldtheoretical nature of nuclear forces, that is, on a purely phenomenological basis. The investigation of this problem forms the subject matter of the present paper.

In view of these remarks, it is evident that such an investigation must largely be concerned with the deter-

\footnotetext{
${ }^{4}$ F. Villars, Phys. Rev. 72, 256 (1947); Helv. Phys. Acta 20 476 (1947). In this connection see also: S. T. Ma and F. C. Yu, Phys. Rev. 62, 118 (1942); C. M $\phi l l e r$ and L. Rosenfeld, Kgl. Danske Vid. Math.-Fys. Medd. Sels. 20, No. 12 (1943); W. Pauli and S. Kusaka, Phys. Rev. 63, 400 (1943).

${ }^{5}$ A. Thellung and F. Villars, Phys. Rev. 73, 924 (1948).
} 
mination of restrictions on the possible form of the solenoidal part of the exchange current density. A number of restrictions are found below to arise from the usual conditions on the Hamiltonian for a system of nucleons of invariance under translation and (both proper and improper) rotations, and of symmetry with respect to all nucleons. The assumptions made in the present treatment are: $(a)$ that only static two-body (but possibly spin-dependent, including tensor) interactions exist between nucleons; (b) that the exchange currents are proportional to the exchange interaction; and $(c)$ that nucleons are spin $\frac{1}{2}$ particles whose states are characterized completely by a position, a spin, and an isotopic spin coordinate. Assumption (a) is probably too stringent in view of the probable existence of many-body ${ }^{6}$ and velocity-dependent forces, but is retained in order to avoid undue complications. Assumption $(b)$ can be somewhat relaxed without great complication and the effects of its relaxation are discussed below (proximity-induced currents). Assumption $(c)$ is necessary in order to impose the condition that the Hamiltonian be symmetric under the exchange of coordinates of any pair of nucleons. To these assumptions should be added: $(d)$ the charge density is assumed to vanish except at the positions of the nucleons. This assumption means that the nucleons are not to be regarded as carriers of a finite-sized charge distribution (as is the case in meson theories where a nucleon carries with it a cloud of charged virtual mesons) but as point charges. While for generality it would be desirable to drop this assumption, we have not done so initially in order to save additional complication of the derived expressions. The effects of dropping this assumption are briefly discussed in Section VIII.

\section{GAUGE INVARIANCE FOR EXCHANGE POTENTIALS}

As is well known, the differential conservation law for charge will be ensured if the Lagrangian function for the system is gauge-invariant. In the particular case of a system of nucleons interacting through exchange and possibly non-exchange) potentials this condition reduces to the condition that the exchange interaction between the nucleons be itself gauge-invariant since all other terms already automatically satisfy this condition or may be made to do so by standard methods. Employing isotopic spin notation, the exchange interaction terms can be written in the form:

$$
H_{x}=\sum_{i, j} V_{i j}\left(\mathbf{r}_{i}-\mathbf{r}_{j}, \boldsymbol{\sigma}_{i}, \boldsymbol{\sigma}_{j}\right)\left(\tau_{i}^{x} \tau_{j}^{x}+\tau_{i}^{y} \tau_{j}^{y}\right),
$$

where $\mathbf{r}_{i}, \boldsymbol{\sigma}_{i}$, and $\boldsymbol{\tau}_{i}$ are, respectively, the position, spin, and isotopic spin operators for the $i$ th nucleon, and $V_{i j}$ is the exchange part of the interaction potential between the $i$ th and $j$ th nucleons (apart from its explicitly indicated isotopic spin dependence) and is a function of the spin operators for the two nucleons and of their spatial separation. Since in the presence of electromag-

\footnotetext{
${ }^{6}$ H. Primakoff and T. Holstein, Phys. Rev. 55, 1218 (1939).
}

netic fields this expression is not gauge-invariant, we are faced with the problem of modifying it properly so that it is gauge-invariant, with the condition that the modified expression reduce to (1) in the absence of electromagnetic fields.

Sachs $^{3}$ solved this problem by assuming Wheeler's representation ${ }^{7}$ of the space-exchange operator as a differential operator and employing the usual method for making a differential operator gauge-invariant. The resultant expression is not unique, but an examination of the method of derivation allows one to write down the general result without difficulty. We shall here give the general result and then demonstrate that it is indeed gauge-invariant. The generalization of (1) which is gauge invariant in the presence of electromagnetic fields can be written

$$
\begin{aligned}
& H_{x}=\sum_{i, j} V_{i j}\left\{\tau _ { i } ^ { P } \tau _ { j } ^ { N } \left[F\left(u_{i j}{ }^{1}, u_{i j}{ }^{2}, \cdots\right)\right.\right. \\
& \left.-i G\left(v_{i j}{ }^{1}, v_{i j}{ }^{2}, \cdots\right)\right] \exp \left[-i e \int \xi_{i j} \cdot \mathbf{A} d \mathbf{x}\right] \\
& +\tau_{i}{ }^{N} \tau_{j}{ }^{P}\left[F\left(u_{i j}{ }^{1}, u_{i j}{ }^{2}, \cdots\right)+i G\left(v_{i j}{ }^{1}, v_{i j}{ }^{2}, \cdots\right)\right] \\
& \left.\quad \times \exp \left[i e \int \xi_{i j} \cdot \mathbf{A} d \mathbf{x}\right]\right\}\left(\tau_{i}{ }^{x} \tau_{j}{ }^{x}+\tau_{i}{ }^{y} \tau_{j}{ }^{y}\right) .
\end{aligned}
$$

Here $e$ is the charge on the proton, $\mathbf{A}=\mathbf{A}(\mathbf{x}, t)$ is the vector potential of the electromagnetic field, $\tau_{i}{ }^{P}$ and $\tau_{i}{ }^{N}$ are the operators

$$
\tau_{i}^{P}=\left(1+\tau_{i}{ }^{Z}\right) / 2, \quad \tau_{i}{ }^{N}=\left(1-\tau_{i}{ }^{Z}\right) / 2,
$$

and $F$ and $G$ are arbitrary real scalar functions of their arguments satisfying only the conditions

$$
F(0,0,0 \cdots)=1, \quad G(0,0,0 \cdots)=0 .
$$

The arguments $u_{i j}{ }^{\alpha}$ and $v_{i j}{ }^{\alpha}$ are defined as

$$
u_{i j}{ }^{\alpha}=e \int \mathbf{A} \cdot \operatorname{curl}_{x} \mathbf{n}_{i j}{ }^{\alpha} d \mathbf{x}, \quad v_{i j}{ }^{\alpha}=e \int \mathbf{A} \cdot \operatorname{curl}_{x} \zeta_{i j}{ }^{\alpha} d \mathbf{x},
$$

where

$$
\begin{array}{r}
\mathbf{n}_{i j}{ }^{\alpha}=\mathfrak{n}^{\alpha}\left(\mathbf{x}-\mathbf{r}_{i}, \mathbf{x}-\mathbf{r}_{j}, \boldsymbol{\sigma}_{i}, \boldsymbol{\sigma}_{j}\right), \\
\zeta_{i j}{ }^{\alpha}=\zeta_{i j}{ }^{\alpha}\left(\mathbf{x}-\mathbf{r}_{i}, \mathbf{x}-\mathbf{r}_{j}, \boldsymbol{\sigma}_{i}, \boldsymbol{\sigma}_{j}\right),
\end{array}
$$

are arbitrary axial vector functions of $\mathbf{x}-\mathbf{r}_{i}, \mathbf{x}-\mathbf{r}_{j}$, and the spin operators of the $i$ th and $j$ th nucleons. The quantity

$$
\xi_{i j}=\xi\left(\mathbf{x}-\mathbf{r}_{i}, \mathbf{x}-\mathbf{r}_{j}, \boldsymbol{\sigma}_{i}, \boldsymbol{\sigma}_{j}\right)
$$

is an arbitrary polar vector function of its indicated arguments satisfying the equation

$$
\operatorname{div}_{x} \xi_{i j}=\delta\left(\mathbf{x}-\mathbf{r}_{j}\right)-\delta\left(\mathbf{x}-\mathbf{r}_{i}\right),
$$

where the functions on the right are three-dimensional Dirac $\delta$-functions.

\footnotetext{
${ }^{7}$ J. A. Wheeler, Phys. Rev. 50, 643 (1936).
} 
Under the gauge transformation

$$
\mathbf{A} \rightarrow \mathbf{A}+\operatorname{grad} \Lambda, \quad \Phi \rightarrow \Phi-\partial \Lambda / \partial t,
$$

the wave function transforms as

$$
\Psi \rightarrow\left\{\prod_{i}\left[\tau_{i}{ }^{P} \exp \left[i e \Lambda\left(\mathbf{r}_{i}\right)\right]+\tau_{i}{ }^{N}\right]\right\} \Psi,
$$

and the expression (2) transforms to

$$
\begin{aligned}
& \left\{\prod_{m}\left(\tau_{m}{ }^{P} \exp \left[-i e \Lambda\left(\mathbf{r}_{m}\right)\right]+\tau_{m}{ }^{N}\right)\right\} \\
& \times \sum_{i, j}\left\{\tau_{i}^{P} \tau_{j}^{N}\left[F\left(u_{i j}{ }^{\alpha}\right)-i G\left(v_{i j}{ }^{\alpha}\right)\right]\right. \\
& \quad \times \exp \left[-i e \int \xi_{i j} \cdot(\mathbf{A}+\operatorname{grad} \Lambda) d \mathbf{x}\right] \\
& +\tau_{i}{ }^{N} \tau_{j}^{P}\left[F\left(u_{i j}{ }^{\alpha}\right)+i G\left(v_{i j}{ }^{\alpha}\right)\right] \\
& \left.\quad \times \exp \left[i e \int \xi_{i j} \cdot(\mathbf{A}+\operatorname{grad} \Lambda) d \mathbf{x}\right]\right\}\left(\tau_{i}{ }^{x} \tau_{j}{ }^{x}+\tau_{i}^{y} \tau_{j}^{y}\right) \\
& \quad \times\left\{\prod_{n}\left[\tau_{n}{ }^{P} \exp \left[i e \Lambda\left(\mathbf{r}_{n}\right)\right]+\tau_{n}{ }^{N}\right)\right\},
\end{aligned}
$$

where use has been made of the fact that the $u_{i j}{ }^{\alpha}$ and $v_{i j}{ }^{\alpha}$ are invariant under the transformation. On commuting either the initial or final factors in braces through the remainder of the expression in (11), one finds easily that (11) resumes the form (2), that is, it is gaugeinvariant, provided

$$
\int \xi_{i j} \cdot \operatorname{grad} \Lambda d \mathbf{x}=\Lambda\left(\mathbf{r}_{i}\right)-\Lambda\left(\mathbf{r}_{j}\right)
$$

But by an integration by parts and use of Eq. (8), one finds immediately that

$$
\int \xi_{i j} \cdot \operatorname{grad} \Lambda d \mathbf{x}=-\int \Lambda \operatorname{div}_{x} \xi_{i j} d \mathbf{x}=\Lambda\left(\mathbf{r}_{i}\right)-\Lambda\left(\mathbf{r}_{j}\right),
$$

establishing the gauge-invariance of (2). It is assumed in the above that $\boldsymbol{n}_{i j}{ }^{\alpha}, \zeta_{i j}{ }^{\alpha}$, and $\xi_{i j}$ fall off sufficiently rapidly for large $\mathbf{x}$ that one may neglect surface integrals arising from integrations by parts.

However, it is not immediately obvious that (2) reduces to (1) in the absence of electromagnetic fields, since in this limit the expression in braces in (2) reduces to $\left(\tau_{i}{ }^{P} \tau_{j}{ }^{N}+\tau_{i}^{N} \tau_{j}{ }^{P}\right)$. However, making use of the fact that the operators

$$
\tau_{i}^{P} \tau_{j}^{P}\left(\tau_{i}^{x} \tau_{j}{ }^{x}+\tau_{i}^{y} \tau_{j}{ }^{y}\right), \quad \tau_{i}{ }^{N} \tau_{j}{ }^{N}\left(\tau_{i}^{x} \tau_{j}{ }^{x}+\tau_{i}^{y} \tau_{j}^{y}\right),
$$

are both identically zero, we may add the quantity $\left(\tau_{i}{ }^{P} \tau_{j}{ }^{P}+\tau_{i}{ }^{N} \tau_{j}{ }^{N}\right)$ inside the braces in (2) without changing its value, and then in the limit as $A$ goes to zero, the expression in braces becomes

$$
\begin{aligned}
&\left\{\tau_{i}{ }^{P} \tau_{j}{ }^{P}+\tau_{i}{ }^{P} \tau_{j}{ }^{N}+\tau_{i}^{N} \tau_{j}{ }^{P}+\tau_{i}{ }^{N} \tau_{j}{ }^{N}\right\} \\
&=\left(\tau_{i}{ }^{P}+\tau_{i}{ }^{N}\right)\left(\tau_{j}{ }^{P}+\tau_{j}{ }^{N}\right)=1,
\end{aligned}
$$

demonstrating the equivalence of (2) and (1) in the absence of electromagnetic fields.
We note further that (2) is Hermitian and that it is invariant under translations and both proper and improper rotations of the coordinate system. However, it is not symmetric under the interchange of the coordinates of any pair of nucleons as it stands, but it becomes so if we add the additional requirement that under the simultaneous interchange of $\mathbf{r}_{i}$ with $\mathbf{r}_{j}$ and $\boldsymbol{\sigma}_{i}$ and $\boldsymbol{\sigma}_{j}:(a)$ The function $F$ is symmetric, (b) The function $G$ is antisymmetric, and (c) The function $\xi_{i j}$ is antisymmetric.

With these conditions (2) appears then to be the most general exchange interaction which is gaugeinvariant and which satisfies the assumptions laid down in Section I.

If $\xi_{i j}$ is written as the sum of its solenoidal and irrotational parts:

$$
\xi_{i j}=\operatorname{grad}_{x} \varphi_{i j}+\operatorname{curl}_{x} \chi_{i j},
$$

we note that we can take $x_{i j}$ to be zero without loss in generality since any solenoidal part of $\xi_{i j}$ can be combined into the function $G$.

\section{THE EXCHANGE CURRENT DENSITY}

If we limit our interest only to those exchange currents flowing in the absence of external electromagnetic fields (that is, neglect the exchange currents which are induced by external fields), then we can expand the exchange interaction (2) in a power series in the vector potential and retain only linear terms. The result of this expansion is

$$
\begin{gathered}
H_{x}=\sum_{i, j} V_{i j}\left\{\left(\tau_{i}{ }^{P} \tau_{j}{ }^{N}+\tau_{i}{ }^{N} \tau_{j}{ }^{P}\right)\right. \\
\times\left[1+e \int \mathbf{A} \cdot \operatorname{curln}_{i j} d \mathbf{x}\right]-i\left(\tau_{i}^{P} \tau_{j}^{N}-\tau_{i}^{N} \tau_{j}^{P}\right) \\
\left.\times\left[e \int \mathbf{A} \cdot\left(\operatorname{curl} \zeta_{i j}+\operatorname{grad} \varphi_{i j}\right) d \mathbf{x}\right]\right\} \\
\times\left(\tau_{i}{ }^{x} \tau_{j}{ }^{x}+\tau_{i}{ }^{y} \tau_{j}{ }^{y}\right),
\end{gathered}
$$

where

$$
\begin{aligned}
\mathbf{n}_{i j} & =\sum_{\alpha}\left(\partial F / \partial u_{i j}{ }^{\alpha}\right)_{A=0} \mathbf{n}_{i j}{ }^{\alpha}, \\
\zeta_{i j} & =\sum_{\alpha}\left(\partial G / \partial v_{i j}{ }^{\alpha}\right)_{A=0} \zeta_{i j}{ }^{\alpha} .
\end{aligned}
$$

The desired operator for the exchange current density may then be obtained from (17) by taking its negative variational derivative with respect to the vector potential with the result:

$$
\begin{aligned}
& \mathbf{j}_{x}=\mathbf{j}_{l}+\mathbf{j}_{t}+\mathbf{j}_{t}^{\prime}, \\
& \mathbf{j}_{l}=i e \sum_{i j} V_{i j}\left(\tau_{i}{ }^{P} \tau_{j}{ }^{N}-\tau_{i}^{N} \tau_{j}{ }^{P}\right) \operatorname{grad} \varphi_{i j}\left({\tau_{i}}^{x} \tau_{j}{ }^{x}+\tau_{i}{ }^{y} \tau_{j}{ }^{\nu}\right) \\
& =e \sum_{i, j} V_{i j}\left(\tau_{i}^{x} \tau_{j}^{y}-\tau_{i}^{y} \tau_{j}^{x}\right) \operatorname{grad} \varphi_{i j}, \\
& \mathbf{j}_{t}=e \sum_{i, j} V_{i j}\left(\tau_{i}{ }^{x} \tau_{j}{ }^{y}-\tau_{i}{ }^{y} \tau_{j}{ }^{x}\right) \operatorname{curl} \zeta_{i j}, \\
& \mathbf{j}_{t}^{\prime}=-c \sum_{i, j} V_{i j}\left(\tau_{i}^{x} \tau_{j}^{x}+\tau_{i}^{y} \tau_{j}^{y}\right) \operatorname{curln}_{i j} \text {. }
\end{aligned}
$$


We shall now discuss these three contributions to the exchange current density separately.

The first of these, $\mathbf{j}_{l}$, obviously represents the irrotational (longitudinal) part of the exchange current density. By combining Eqs. (8) and (16) we obtain the equation

$$
\nabla^{2} \varphi_{i j}=\delta\left(\mathbf{x}-\mathbf{r}_{j}\right)-\delta\left(\mathbf{x}-\mathbf{r}_{i}\right),
$$

with the unique solution (which vanishes at infinity):

$$
\varphi_{i j}=1 / 4 \pi\left[\left(1 /\left|\mathbf{x}-\mathbf{r}_{i}\right|\right)-\left(1 /\left|\mathbf{x}-\mathbf{r}_{j}\right|\right)\right] .
$$

Hence the irrotational part of the current density is completely determined by the condition of differential charge conservation, as would be expected. The contribution to this part of the current from each pair of interacting nucleons has the simple form of potential flow between a source and sink of charge of equal strength located at the positions of the nucleons. Conjugate pairs of nuclei are defined as those pairs having identical wave functions except for the interchange of neutrons and protons. Since the isotopic spin factor in the irrotational part of the exchange current density changes sign under an interchange of neutrons and protons in a nuclear wave function, we see that expectation values (diagonal matrix elements) of the irrotational exchange current density are equal but opposite in sign for two conjugate nuclei and therefore vanish for self-conjugate nuclei. It is important to note that this theorem is true only for the diagonal matrix elements; the fact that it is not true for the off-diagonal elements (existence of exchange current fluctuations) shows that an interaction of the irrotational currents with the electromagnetic field is still possible for self-conjugate nuclei (such as the deuteron) even though their average value is zero.

Turning now to the solenoidal (transverse) part of the exchange current density given by the two terms $\mathbf{j}_{t}$ and $\mathbf{j}_{t}{ }^{\prime}$, we see that the two terms are similar except for their different isotopic spin dependence. The isotopic spin dependence of the first is the same as that for the longitudinal part of the current and the same remarks as made above are therefore applicable to it. The second term, however, does not change sign under an interchange of protons and neutrons in a nucleus and hence gives equal expectation values for conjugate nuclei and a non-vanishing expectation value for self-conjugate nuclei. The significance of this fact will be discussed more fully below.

These solenoidal parts of the current distribution are not determined by the condition of charge conservation. The only conditions to which they are subject are those resulting as a consequence of the discussion near the end of the last section. These require that $\zeta_{i j}$ be an axial vector function of $\mathbf{R}_{i}=\mathbf{x}-\mathbf{r}_{i}, \mathbf{R}_{j}=\mathbf{x}-\mathbf{r}_{j}, \boldsymbol{\sigma}_{i}$, and $\boldsymbol{\sigma}_{j}$, which is antisymmetric under the simultaneous interchange of $\mathbf{R}_{i}$ with $\mathbf{R}_{j}$ and $\boldsymbol{\sigma}_{i}$ with $\boldsymbol{\sigma}_{j}$; and that $\boldsymbol{n}_{i j}$ be an axial vector function of the same variables but symmetric under the interchange above. However, before discussing the exchange current density in more detail, we will first discuss the operators for the exchange contribution to the magnetic moment of a nucleus.

\section{EXCHANGE MAGNETIC MOMENTS}

From the expression for the exchange current density, one can readily calculate the operator for the exchange magnetic moment of a nucleus. This also breaks naturally into three parts:

$$
\begin{aligned}
& \mathbf{M}_{x}=\mathbf{M}_{l}+\mathbf{M}_{t}+\mathbf{M}_{t}^{\prime}, \\
& \mathbf{M}_{l}=\frac{1}{2} e \sum_{i, j} V_{i j}\left(\tau_{i}^{x} \tau_{j}^{y}-\tau_{i}^{y} \tau_{j}{ }^{x}\right) \int \mathbf{x} \times \operatorname{grad} \varphi_{i j} d \mathbf{x} \\
& =-\frac{1}{2} e \sum_{i, j} V_{i j}\left(\tau_{i}^{x} \tau_{j}^{y}-\tau_{i}^{y} \tau_{j}^{x}\right)\left[\mathbf{r}_{i} \times \mathbf{r}_{j}\right], \\
& \mathbf{M}_{t}=\frac{1}{2} e \sum_{i, j} V_{i j}\left(\tau_{i}^{x} \tau_{j}^{y}-\tau_{i}^{y} \tau_{j}^{x}\right) \int \mathbf{x} \times \operatorname{curl} \zeta_{i j} d \mathbf{x} \\
& =\frac{1}{2} e \sum_{i, j} V_{i j}\left(\tau_{\imath}{ }^{x} \tau_{j}^{y}-\tau_{i}^{y} \tau_{j}^{x}\right) \mathbf{Z}_{i j}, \\
& \mathbf{M}_{t}^{\prime}=-\frac{1}{2} e \sum_{i, j} V_{i j}\left(\tau_{i}{ }^{x} \tau_{j}{ }^{x}+\tau_{i}^{y} \tau_{j}^{y}\right) \int \mathbf{x} \times \operatorname{curln}_{i j} d \mathbf{x} \\
& =-\frac{1}{2} e \sum_{i, j} V_{i j}\left(\tau_{i}^{x} \tau_{j}^{x}+\tau_{i}^{y} \tau_{j}^{y}\right) \mathbf{H}_{i j}, \\
& \mathbf{Z}_{i j}=\mathbf{Z}\left(\mathbf{r}_{i}-\mathbf{r}_{j}, \boldsymbol{\sigma}_{i}, \boldsymbol{\sigma}_{j}\right)=\int \mathbf{x} \times \operatorname{curl} \zeta_{i j} d \mathbf{x}=\int \zeta_{i j} d \mathbf{x}, \\
& \mathbf{H}_{i j}=\mathbf{H}\left(\mathbf{r}_{i}-\mathbf{r}_{j}, \sigma_{i}, \sigma_{j}\right)=\int \mathbf{x} \times \operatorname{curl}_{i j} d \mathbf{x}=\int \mathbf{n}_{i j} d \mathbf{x} .
\end{aligned}
$$

The first part, arising from the irrotational part of the current, is the same as that calculated by $\mathrm{Sachs}^{3}$ who also evaluated its expectation value for $\mathrm{H}^{3}$ and $\mathrm{He}^{3}$ and found it to be of insufficient magnitude to explain the experimental results for these nuclei. The magnitudes of the remaining two parts depend, of course, on the form of the arbitrary functions occurring in them, but a considerable amount of information can nevertheless be obtained concerning them as a result of the remaining conditions on the arbitrary functions.

Consider first the quantity $V_{i j} Z_{i j}$. The conditions of translational and rotational invariance, and symmetry of the Hamiltonian in all nucleons, requires that it be an axial vector function only of the vector separation of the $i$ th and $j$ th nucleons, $\mathbf{r}_{i j}=\mathbf{r}_{i}-\mathbf{r}_{j}$, and of the spin operators for the two nucleons, and that it be antisymmetric under the exchange of the coordinates of the two nucleons. Now, apart from a multiplying scalar function of the distance between the two nucleons, the only axial vectors which can be constructed from the polar vector $\mathbf{r}_{i j}$ and the two axial vectors $\boldsymbol{\sigma}_{i}$ and $\boldsymbol{\sigma}_{j}$ and which are antisymmetric under the interchange of coordinates of the two nucleons are the four quantities (see Appendix):

$$
\begin{gathered}
\boldsymbol{\sigma}_{i}-\boldsymbol{\sigma}_{j}, \\
{\left[\mathbf{r}_{i j} \cdot\left(\boldsymbol{\sigma}_{i}-\mathbf{\sigma}_{j}\right)\right] \mathbf{r}_{i j},} \\
\boldsymbol{\sigma}_{i} \times \boldsymbol{\sigma}_{j}, \\
\left(\mathbf{r}_{i j} \cdot \boldsymbol{\sigma}_{\imath} \times \boldsymbol{\sigma}_{j}\right) \mathbf{r}_{i j} .
\end{gathered}
$$


The most general form which $V_{i j} Z_{i j}$ can take is therefore a linear-combination of these four quantities multiplied by arbitrary functions of the distance between the two nucleons.

On the other hand, the conditions on $V_{i j} \mathrm{H}_{i j}$ are the same as those on $V_{i j} \mathbf{Z}_{i j}$ except for the fact that this quantity must be symmetric under the interchange of space and spin coordinates of the pair of nucleons. There are only three such axial vectors (apart from an arbitrary multiplying scalar function of the distance between the nucleons):

(e)

$$
\begin{gathered}
\boldsymbol{\sigma}_{i}+\boldsymbol{\sigma}_{j}, \\
\left.\mid \mathbf{r}_{i j} \cdot\left(\boldsymbol{\sigma}_{i}+\boldsymbol{\sigma}_{j}\right)\right] \mathbf{r}_{i j}, \\
\left(\mathbf{r}_{i j} \cdot \boldsymbol{\sigma}_{i}\right)\left[\mathbf{r}_{i j} \times \boldsymbol{\sigma}_{j}\right]+\left(\mathbf{r}_{i j} \cdot \boldsymbol{\sigma}_{j}\right)\left[\mathbf{r}_{i j} \times \boldsymbol{\sigma}_{i}\right] .
\end{gathered}
$$

The most general form of spin dependence of the magnetic moment operator is therefore determined by group-theoretical principles combined with the plausible symmetry conditions which we have imposed on the Hamiltonian. It is of interest to note that the existence of contributions to the magnetic moments arising from solenoidal exchange currents is consequent on the nucleons having spin. For spinless nucleons the exchange moment is completely determined by the irrotational exchange currents which in turn are completely specified by the requirement of gauge invariance.

The forms (a), (b), (e) and (f) which are linear in the nucleon spins, can be given a very simple physical interpretation. In the Hamiltonian, the forms (a) and (e) lead to terms having the form of the interaction of the magnetic moment of a nucleon with the magnetic field but for which the magnitude of the magnetic moment is a function of the spatial separation of this nucleon from the other nucleons present. Hence these terms correspond to a modification of the intrinsic magnetic moment of a nucleon by the presence of other nucleons in its neighborhood, that is, to a proximity effect on the intrinsic magnetic moments of nucleons. The forms (b) and (f) are similar except that they involve a modification of that component of the intrinsic magnetic moment of a nucleon parallel to the line joining the nucleon and its disturbing neighbor. Terms of this character if actually present will lead to nonadditivity of the neutron and proton magnetic moments in the deuteron.

\section{COMPARISON WITH MESON THEORY}

It is interesting to compare the results obtained in the preceding section with those obtained by fieldtheoretical calculations on the basis of the exchange of mesons as the origin of exchange currents. ${ }^{4}$ In such calculations as so far carried out, the exchange moment is found to be a combination of the longitudinal contribution (25) together with contributions of the form (30-c, d). The absence in meson theory of the other possible forms which we have found in the phenomeno- logical theory is easily explained when one remembers that magnetic moment calculations in meson theory have been carried out only to the lowest non-vanishing order in $e$ and in the meson coupling constant $g$ (that is, to order $g^{2} e$ ). To this approximation there is a symmetry between positive and negative mesons and neutrons and protons such that the interchange of a neutron and proton causes a reversal in sign of the exchange currents and therefore of the magnetic moments. Hence, one cannot expect to obtain terms of the form (31-e, f, g) in a meson theory calculation which does not go beyond the order $g^{2} e$. Furthermore, meson theory calculations, to this order, while allowing one to obtain the meson contribution to the intrinsic magnetic moment of an isolated nucleon, still cannot account for any modification of this intrinsic moment by the proximity of another nucleon which is an essentially higher order effect. This rules out obtaining terms of the form $(30-a, b)$ in a second order meson theory calculation.

It might be assumed that our phenomenological theory is of sufficient generality to contain all results (except for the form of the arbitrary functions which occur in our expressions) which could be obtained from any meson theory. This is not quite the case, for the following reason. We have assumed in our theory (assumption $(d)$ ) that while currents may flow in the intervening space between nucleons, the electric charge density vanishes everywhere except at the positions of the nucleons. This is not actually the case in meson theory. As a result of the emission and re-absorption of virtual mesons by a nucleon, there is a finite charge density in the region surrounding a nucleon of radius equal to the Compton wave-length of the meson in order of magnitude. It is this fact, for example, which is presumably responsible for the observed scattering of slow neutrons by electrons. ${ }^{8}$ The existence of this charge density over a finite region does not influence the results obtained for the exchange magnetic moment in second-order meson theory, but will be of importance in higher orders. The effect in our phenomenological theory of having a finite distribution of charge about nucleons is discussed briefly in a later section.

\section{SPIN DEPENDENCE OF THE EXCHANGE CURRENT DENSITY}

Returning again to the consideration of the operator for the exchange current density we note that by the application of the same methods as employed above for the magnetic moment operator we may find the possible forms of spin dependence of the solenoidal part of the exchange current. The two arbitrary functions $\zeta_{i j}$ and $\boldsymbol{n}_{i j}$ in Eqs. (20) and (21) must be axial vector functions formed from the four vectors $\mathbf{R}_{i}=\mathbf{x}-\mathbf{r}_{i}$,

${ }^{8}$ Havens, Rabi, and Rainwater, Phys. Rev. 72, 634 (1947); Fermi and Marshall, Phys. Rev. 72, 1139 (1947); Rainwater, Rabi and Havens, Phys. Rev. 75, 1295 (1948); Slotnick and Heitler, Phys. Rev. 75, 1645 (1949); K. M. Case, Phys. Rev. 76, 1 (1949); Dancoff and Drell, Phys. Rev. 76, 205 (1949). 
$\mathbf{R}_{j}=\mathbf{x}-\mathbf{r}_{j}, \boldsymbol{\sigma}_{i}$ and $\boldsymbol{\sigma}_{j}$, and must be antisymmetric and symmetric, respectively, under the simultaneous interchange of $\mathbf{R}_{i}$ with $\mathbf{R}_{j}$ and $\boldsymbol{\sigma}_{i}$ with $\boldsymbol{\sigma}_{j}$. One finds without difficulty (see Appendix) that apart from multiplying scalar functions formed from $\mathbf{R}_{i}$ and $\mathbf{R}_{j}$, there are only 27 linearly independent axial vectors which may be formed from the four vectors listed above. There are:

$$
\begin{aligned}
\left(\mathbf{R}_{2} \cdot \boldsymbol{\sigma}_{2}\right)\left[\mathbf{R}_{1} \times \boldsymbol{\sigma}_{1}\right]+\left(\mathbf{R}_{2} \cdot \boldsymbol{\sigma}_{1}\right)\left[\mathbf{R}_{1} \times \boldsymbol{\sigma}_{2}\right] & +\left(\mathbf{R}_{1} \cdot \boldsymbol{\sigma}_{2}\right)\left[\mathbf{R}_{2} \times \boldsymbol{\sigma}_{1}\right]+\left(\mathbf{R}_{1} \cdot \boldsymbol{\sigma}_{1}\right)\left[\mathbf{R}_{2} \times \boldsymbol{\sigma}_{2}\right] \\
+ &
\end{aligned}
$$

$$
\left(\mathbf{R}_{2} \cdot \sigma_{2}\right)\left(\mathbf{R}_{1} \cdot \sigma_{1}\right)\left[\mathbf{R}_{1} \times \mathbf{R}_{2}\right]
$$$$
\text { (p) }\left(\sigma_{1} \cdot \mathbf{R}_{1} \times \mathbf{R}_{2}\right)\left[\mathbf{R}_{1} \times \mathbf{R}_{2}\right] \pm\left(\sigma_{2} \cdot \mathbf{R}_{1} \times \mathbf{R}_{2}\right)\left[\mathbf{R}_{1} \times \mathbf{R}_{2}\right] \text {, }
$$$$
\text { (q) }\left\{\left(\mathbf{R}_{1} \cdot \sigma_{1}\right)\left(\mathbf{R}_{1} \cdot \boldsymbol{\sigma}_{2}\right) \pm\left(\mathbf{R}_{2} \cdot \boldsymbol{\sigma}_{1}\right)\left(\mathbf{R}_{2} \cdot \boldsymbol{\sigma}_{2}\right)\right\}\left[\mathbf{R}_{1} \times \mathbf{R}_{2}\right] \text {. }
$$

All of these are either symmetrical or antisymmetrical under the interchanges mentioned above; hence by multiplying each of these by an arbitrary symmetrical or antisymmetrical scalar function formed from the vectors $\mathbf{R}_{i}$ and $\mathbf{R}_{j}$, taking appropriate linear combinations of the results for $\zeta_{i j}$ and $\boldsymbol{n}_{i j}$, and substituting in Eqs. (20) and (21), we obtain the most general form for the solenoidal part of the exchange currents. The total number of terms will be reduced somewhat when the curl operations in (20) and (21) are explicitly carried out, ${ }^{9}$ but since there are no immediate applications for these results, we have not carried the reduction any further.

It will be noted that if one had spinless nucleons then only one solenoidal term (32-a) would survive. This

\footnotetext{
9 That the number of terms is reduced follows from the fact that (20) and (21) represent polar vectors constructed from $\mathbf{R}_{i}, \mathbf{R}_{j}$, $\boldsymbol{\sigma}_{i}$ and $\boldsymbol{\sigma}_{j}$. One finds that apart from scalar multiplying factors there are at most 24 polar vectors which may be constructed from those above. However, since these must be solenoidal vectors, one must add the condition that the divergence of these expressions vanishes identically which may further cut down the number of independent terms.
}

term corresponds to a current distribution which is rotationally symmetric about the line joining the two nucleons and for which the direction of flow of the current at any point lies always in the plane containing the point and the two nucleons. Such a current distribution obviously gives rise to no resultant magnetic moment.

Again comparing our results with those to be expected from a lowest order meson theory calculation, we note that for the same reasons as given above in regard to the magnetic moment operator we would not expect any terms symmetric under the interchange of the pair of interacting nucleons so that no contribution of the form (21) would appear, and we would not expect any terms linear in the spin of the nucleons in (20).

\section{PROXIMITY-INDUCED CURRENTS}

It is apparent from what has been said above that the exchange of charge between two nucleons interacting under the influence of an exchange force is actually effected only by the irrotational part of the exchange current density. The solenoidal part of the latter simply describes the flow of currents in closed loops induced by some mechanism when two nucleons are in proximity. The term "exchange currents" applied to the solenoidal part of the current density is therefore somewhat of a misnomer and "proximity-induced currents" would be perhaps a more appropriate designation. We mention this fact because on a purely phenomenological basis there is no a priori reason for assuming (assumption $(b)$ ) that such proximity-induced solenoidal currents do not flow even about nucleons interacting under an ordinary force. In fact such currents would be expected to flow about two neutrons or two protons is conventional meson theory in higher order where pairs of oppositely charged mesons are exchanged. The possible forms which the current density and magnetic moment operators may take in such cases may be calculated by methods essentially identical with those used above.

\section{EFFECT OF FINITE NUCLEONIC CHARGE DISTRIBUTION}

We shall in this section describe briefly the effect on our theory of the existence of finite charge distributions about nucleons such as those connected with the occurrence of a cloud of virtual charged mesons about a nucleon. In our non-relativistic approximation one can regard such a finite charge distribution as being rigidly bound to a nucleon. The specific form of this charge cloud will depend on whether the nucleon is a proton or neutron and hence will involve $\tau^{Z}$. The direct interaction of the nucleon with the electromagnetic field will then not depend only on the value of the field quantities at the position of the nucleon but will involve integrals of the field over the charge distribution. This means that under the gauge transformation (9), the wave function will not transform according to (10) but 
will involve instead in each term of the parenthesis in (10), an exponential of an integral of the gauge function $\Lambda$ over the charge distribution for proton or neutron. The condition for the gauge invariance of the exchange interaction term will then require that the function $\xi_{i j}$ satisfy not Eq. (8) but another equation in which the $\delta$-functions are replaced by finite source functions, viz. :

$$
\begin{aligned}
\operatorname{div} \xi_{i j}=\left[\rho_{P}\left(\mathbf{x}-\mathbf{r}_{j}\right)-\rho_{N}\left(\mathbf{x}-\mathbf{r}_{j}\right)\right] & \\
- & {\left[\rho_{P}\left(\mathbf{x}-\mathbf{r}_{i}\right)-\rho_{N}\left(\mathbf{x}-\mathbf{r}_{i}\right)\right], } \\
\int \rho_{P}(\mathbf{x}) d \mathbf{x} & =1, \quad \int \rho_{N}(\mathbf{x}) d \mathbf{x}=0,
\end{aligned}
$$

where $\rho_{P}$ is the charge distribution function for the proton and $\rho_{N}$ is the charge distribution function for the neutron. The net result is to leave our expression for the solenoidal exchange current the same but to modify the irrotational exchange current density and its contribution to the magnetic moment in accordance with the change (33).

\section{SUMMARY AND CONCLUSIONS}

We have shown above that one can describe in a phenomenological theory the effects of electric currents induced by the interaction of two nucleons in the nonrelativistic approximation (including neglect of velocitydependent interactions) with two-body forces. In such a theory it is possible to account for: (1) "true" (irrotational) exchange currents, (2) proximity-induced (solenoidal) currents, (3) proximity-induced changes in the intrinsic magnetic moments of nucleons (non-additivity of moments). The effects of a finite charge distribution in nucleons can be included in the theory.

A generalization to include many-body forces can probably be carried through by the same methods as used above, but with a considerable increase in complication. To include velocity-dependent forces and relativistic effects would, however, probably require major changes in methodology.

The shortcomings of a phenomenological approach to exchange currents lies in the fact that there is still considerable arbitrariness in the theory, only the irrotational exchange current density being fixed by the theory. The calculations ${ }^{4}$ made on the basis of partticular models for the exchange of charge (meson theories) have demonstrated that the results obtained do depend rather critically on the particular model assumed. Under these circumstances any further developments in the phenomenological theory must be based on experimental results.

\section{APPENDIX}

In the exposition above, we are faced with the problems (1) of finding the most general axial vector which can be formed from two spin vectors (say $\boldsymbol{\Sigma}$ and $\boldsymbol{\sigma}$ ) and a (polar) position vector (say R), and (2) of finding the most general axial vector which may be constructed from two spin vectors $(\boldsymbol{\Sigma}, \boldsymbol{\sigma})$ and two (polar) position vectors (say $\mathbf{R}$ and $\mathbf{r}$ ). Each of these may obviously be written as a linear combination of all possible linearly independent axial vectors which may be constructed from the vectors enumerated above. Furthermore, each of these linearly independent axial vectors can be factored into an arbitrary scalar function of the position vector or vectors and a purely angle-dependent factor which has the required transformation properties. For the purposes of finding the latter we may consider the position vectors $\mathbf{R}$ and $\mathbf{r}$ as being of unit magnitude.

Since the spin vectors transform under both proper and improper rotations as antisymmetric tensors of the second rank, it is useful to represent them as two index quantities, viz:

$$
\mathbf{\sigma} \rightarrow \sigma_{\mu \nu}=-\sigma_{\nu \mu}, \quad \mathbf{\Sigma} \rightarrow \Sigma_{\mu \nu}=-\Sigma_{\nu \mu},
$$

where we shall use the arrow to designate the process of changing the representation from tensor representation to vector representation, or vice versa. Since the spin vector components satisfy the algebra:

$$
\begin{array}{rlrl}
\sigma_{x}{ }^{2} & =\sigma_{y}{ }^{2}=\sigma_{z}{ }^{2}=1, & \Sigma_{x}{ }^{2}=\Sigma_{y}{ }^{2}=\Sigma_{z}{ }^{2}=1, \\
\sigma_{x} \sigma_{y} & =i \sigma_{z}, & \Sigma_{x} \Sigma_{y}=i \Sigma_{z}, \\
\sigma_{z} \sigma_{x} & =i \sigma_{y}, & & \Sigma_{z} \Sigma_{x}=i \Sigma_{y}, \\
\sigma_{y} \sigma_{z} & =i \sigma_{x}, & \Sigma_{y} \Sigma_{z}=i \Sigma_{x},
\end{array}
$$

any expression of higher than first degree in the components of either spin vector can always be reduced to one of first degree. Thus the only tensors which can be formed from the spin vectors (including the scalar, unity) are:

$\left.\begin{array}{cl}1 & \text { Scalar with } 1 \text { component. } \\ \sigma_{\mu \nu} \\ \Sigma_{\mu \nu}\end{array}\right\} \quad \begin{aligned} & \text { Antisymmetric second rank tensors } \\ & \text { with } 3 \text { linearly independent com- } \\ & \text { ponents each. } \\ & \sigma_{\mu \alpha} \Sigma_{\alpha \nu} \\ & \text { Second rank tensor with } 9 \text { linearly } \\ & \text { independent components. }\end{aligned}$

These 16 quantities may be rearranged to form the more convenient tensors:

$$
\begin{aligned}
& \left.\begin{array}{c}
1 \\
\sigma_{\alpha \beta} \Sigma_{\beta \alpha} \rightarrow(\boldsymbol{\sigma} \cdot \mathbf{\Sigma})
\end{array}\right\} \quad \text { Two scalars each with } 1 \text { component. } \\
& \sigma_{\mu \nu} \\
& \left.\begin{array}{c}
\Sigma_{\mu \nu} \\
\sigma_{\mu \alpha} \Sigma_{\alpha \nu}-\sigma_{\nu \alpha} \Sigma_{\alpha \mu} \rightarrow \boldsymbol{\sigma} \times \mathbf{\Sigma}
\end{array}\right\} \begin{array}{l}
\text { Antisymmetric second rank tensors } \\
\text { with } 3 \text { linearly independent com- } \\
\text { ponents each. }
\end{array} \\
& \frac{3}{2}\left\{\sigma_{\mu \alpha} \Sigma_{\alpha \nu}+\sigma_{\nu \alpha} \Sigma_{\alpha \mu}\right\} \quad \text { Second rank symmetric traceless tensor } \\
& -\delta_{\mu \nu}\left\{\sigma_{\alpha \beta} \Sigma_{\beta \alpha}\right\} \quad \text { with } 5 \text { linearly independent com- }
\end{aligned}
$$

From the one vector $\mathbf{R} \rightarrow R_{\alpha}$ we can form the series of tensors:

$$
1, R_{\alpha}, R_{\alpha} R_{\beta}, \cdots, R_{\alpha} R_{\beta} \cdots R_{\gamma} \cdots, \cdots .
$$

If $\mathbf{R}$ is considered of unit magnitude, then any contraction on indices leads only to another tensor appearing earlier in the list.

Now antisymmetrical second rank tensors can be formed from the vector $\mathbf{R}$ and the two spin vectors in the following ways:

I. By multiplying the scalars $1,(\boldsymbol{\sigma} \cdot \mathbf{\Sigma})$ with an antisymmetric second rank tensor formed from the $R$ 's. No such exist.

II. By multiplying the antisymmetric second rank tensors $\sigma_{\mu \nu}$, $\Sigma_{\mu \nu}, \sigma_{\mu \beta} \Sigma_{\beta \nu}-\sigma_{\nu \beta} \Sigma_{\beta \mu}$ by (A) the scalar 1, yielding:

$$
\begin{aligned}
& \text { A. 1. } \sigma_{\mu \nu} \rightarrow \boldsymbol{\sigma} \text {, } \\
& \text { 2. } \Sigma_{\mu \nu} \rightarrow \Sigma \\
& \text { 3. } \sigma_{\mu \beta} \Sigma_{\beta \nu}-\sigma_{\nu \beta} \Sigma_{\beta \mu} \rightarrow \boldsymbol{\sigma} \times \Sigma \text {, }
\end{aligned}
$$

or (B) by the second rank tensor $R_{\alpha} R_{\nu}$, contracting on one index and antisymmetrizing on the remaining index, yielding:

B. 1. $\sigma_{\mu \alpha} R_{\alpha} R_{\nu}-\sigma_{\nu \alpha} R_{\alpha} R_{\mu} \rightarrow(\boldsymbol{\sigma} \cdot \mathbf{R}) \mathbf{R}$.

2. $\Sigma_{\mu \alpha} R_{\alpha} R_{\nu}-\Sigma_{\nu \alpha} R_{\alpha} R_{\mu} \rightarrow(\mathbf{\Sigma} \cdot \mathbf{R}) \mathbf{R}$.

$$
\begin{aligned}
& \text { 3. }\left[\sigma_{\mu \beta} \Sigma_{\beta \alpha}-\sigma_{\alpha \beta} \Sigma_{\beta \mu}\right] R_{\alpha} R_{\nu} \\
& -\left[\sigma_{\nu \beta} \Sigma_{\beta \alpha}-\sigma_{\alpha \beta} \Sigma_{\beta \mu}\right] R_{\alpha} R_{\mu} \rightarrow(\mathbf{R} \cdot \boldsymbol{\sigma} \times \mathbf{\Sigma}) \mathbf{R} .
\end{aligned}
$$

III. By multiplying the symmetric traceless tensor,

$$
\frac{3}{2}\left[\sigma_{\mu \beta} \Sigma_{\beta \alpha}+\sigma_{\alpha \beta} \Sigma_{\beta \mu}\right]-\delta_{\mu \alpha}\left(\sigma_{\beta \gamma} \Sigma_{\gamma \beta}\right)
$$


by the second rank tensor $R_{\alpha} R_{\nu}$, contracting on one index, and antisymmetrizing on the remaining indices:

$$
\begin{aligned}
\left\{\frac{3}{2}\left[\sigma_{\mu \beta} \Sigma_{\beta \alpha}+\sigma_{\alpha \beta} \Sigma_{\beta \mu}\right]-\delta_{\mu \alpha}\left(\sigma_{\beta \gamma} \Sigma_{\gamma \beta}\right)\right\} R_{\alpha} R_{\nu} \\
-\left\{\frac{3}{2}\left[\sigma_{\nu \beta} \Sigma_{\beta \alpha}+\sigma_{\alpha \beta} \Sigma_{\beta \nu}\right]-\delta_{\nu \alpha}\left(\sigma_{\beta \gamma} \Sigma_{\gamma \beta}\right)\right\} R_{\alpha} R_{\mu} \\
\rightarrow \frac{3}{2}\{(\mathbf{R} \cdot \boldsymbol{\sigma})[\mathbf{R} \times \mathbf{\Sigma}]+(\mathbf{R} \cdot \mathbf{\Sigma})[\mathbf{R} \times \boldsymbol{\sigma}]\} .
\end{aligned}
$$

We thus obtain at most 7 linearly independent axial vectors formed from two spin vectors and a unit polar vector.
The process for finding the totality of linearly independent axial vectors from two spin vectors and two unit polar vectors is practically identical. One can now form more tensors from the polar vectors, viz.:

$$
1, R_{\alpha}, r_{\alpha}, R_{\alpha} R_{\beta}, R_{\alpha} r_{\beta}, r_{\alpha} r_{\beta} \text {, etc. }
$$

By multiplication and contraction with the tensors formed from the spin vectors one then finds the 27 linearly independent axial vectors given in the text.

\title{
Diffusion in the Ionosphere
}

\author{
M. H. Johnson and E. O. Hulburt \\ Naval Research Laboratory, Washington, D. C.
}

(Received April 12, 1950)

\begin{abstract}
Diffusion is treated by showing that the action of a medium on a diffusing gas is that of a dissipative force. When the theory is applied to an electrically neutral ionic gas in a gravitational field it is found that the mixture of positive and negative ions diffuses as a single gas because of the electrical polarization charges within the ionic cloud. In the presence of a magnetic field, the diffusion cannot be expressed in terms of the ionic density until the electrodynamical equations governing the flow of electrical current have been explicitly solved. Solutions are obtained for special cases which show that a strong magnetic field completely inhibits the diffusion due to concentration gradients in the transverse plane and has little effect on the diffusion due to the gravitational force.
\end{abstract}

\section{INTRODUCTION}

$T$ $\mathrm{HE}$ role played by diffusion in the formation of ion banks in the upper ionosphere ( $F$-region) is not settled in our opinion because no one has given an adequate treatment of the diffusion processes. Too little attention has been paid to the electrical polarization developed by the diffusion current and to the reaction of the resulting electric field on the diffusion, although the existence of these effects has been recognized., 2

Transport phenomena are usually treated by the kinetic molecular theory which yields a distribution function for the different kinds of molecules. No one has yet explicitly formulated the diffusion equations for a three component mixture (positive ions, negative ions or electrons, neutral molecules), because of the inherent mathematical complexity of the kinetic molecular theory.

In the present paper this difficulty is avoided by introducing the concept of a dissipative force (Section II). The definition of the diffusion coefficient and the ideal gas law lead directly to a force equation which shows that the pressure gradient in a diffusing gas is balanced by a force proportional to the diffusion velocity. This force acts whenever a diffusion current flows. The diffusion current can then be obtained in any field of force from the balance of all the forces acting on the diffusing gas. In this way the kinetic molecular theory enters into the determination of the mass motion only through the diffusion coefficient and the ideal gas law.

${ }^{1}$ E. O. Hulburt, Phys. Rev. 34, 1167 (1929).

2 T. G. Cowling, M.N.R.A.S. 93, 90 (1932).
A comparatively simple treatment of the migration of equal numbers of positive and negative ions through a neutral gas in a gravitational field is possible with the concept of dissipative force (Section III). It is shown that the mixture diffuses as a single gas whose diffusion coefficient, in case the negative ions are electrons, is equal to twice the diffusion coefficient of the positive ions and whose scale height is given by the average molecular weight of the ions. An electrical field exists throughout the ionic cloud which is derived from internal polarization charges. It is this electric field which binds the motion of the positive and negative ions together, thereby making it possible to describe the mixture as a single gas.

The treatment is extended to include the effect of a magnetic field (Section IV). The diffusion now depends on the force exerted by the magnetic field on any electrical currents which may be present. An exact solution is obtained for a constant diffusion coefficient and constant magnetic field which shows that a circulation of electrical current in the plane perpendicular to the magnetic field must take place in such a way that the diffusion due to pressure gradients becomes negligible for conditions in the ionosphere. The diffusion current due to gravity is unchanged and is accompanied by an electric field, derived from polarization charges, which is perpendicular to the magnetic and gravitational fields. In case the diffusion coefficient is not constant, additional electrical currents flow in such a way that the gravitational diffusion current of most of the ionic cloud is characterized by the diffusion coefficient at a certain median altitude. Thus the magnetic 